\title{
Effect of humidified versus nonhumidified CPAP on inflammatory response and nasopharyngeal symptoms in healthy participants
}

\author{
Hajed M. Al-Otaibi, PhD', Mohammed D. Alahmari, PhD², Thekra N. Al-Maqati, MSc ${ }^{3}$, Abdullah Ghazwani, MRes ${ }^{2}$
}

\begin{abstract}
HM Al-Otaibi, MD Alahmari, TN Al-Maqati, A Ghazwani. Effect of humidified versus nonhumidified CPAP on inflammatory response and nasopharyngeal symptoms in healthy participants. Can J Respir Ther 2020;56:21-24. doi: 10.29390/cjrt-2020-005.

Introduction: Continuous positive airway pressure (CPAP) may induce nasal inflammation because of mucosal compression or dryness. This study examined the impact of humidified versus nonhumidified CPAP on nasal inflammation and upper airway symptoms.

Methods: Seventeen healthy male subjects with no previous or current history of nasal symptoms were recruited. All subjects underwent 3 hours of nonhumidified CPAP at $12.5 \mathrm{cmH}_{2} \mathrm{O}$ via nasal mask. Among the 17 studied subjects, seven returned to receive a humidified $\mathrm{CPAP}$ at $12.5 \mathrm{cmH} \mathrm{O}_{2} \mathrm{O}$ via nasal mask. The nasal wash leukocyte count was assessed at baseline and after each CPAP setting. The white blood cell (WBC) count and levels of WBCs that are mononuclear cells (including lymphocytes and monocytes) were monitored. A six-point nasal score was also assessed before and after the CPAP intervention.

Results: The nasal wash WBC count $\left(10^{3} / \mu \mathrm{L}\right)$ and mononuclear cell level $\left(10^{3} / \mu \mathrm{L}\right)$ at baseline, on $12.5 \mathrm{cmH}_{2} \mathrm{O}$ humidified $\mathrm{CPAP}$, and on $12.5 \mathrm{cmH}_{2} \mathrm{O}$ nonhumidified CPAP were significantly different $(p=0.016 ; p=0.003)$. Changes in nasopharyngeal symptoms occurred in 12 of 17 subjects $(70.5 \%)$ in the nonhumidified group. Participants experienced at least one nasal symptom after application of nonhumidified $\mathrm{CPAP}_{\text {at }} 12.5 \mathrm{cmH}_{2} \mathrm{O}$.

Conclusion: The present investigation suggests that humidified CPAP was not associated with early nasal inflammation and there were fewer nasopharyngeal symptoms. Further study is required to confirm the results and evaluate the impact of adding heat to the humidified CPAP system.
\end{abstract}

Key Words: CPAP; humidification; nasal inflammation; respiratory symptoms; inflammatory markers

\section{INTRODUCTION}

Continuous positive airway pressure (CPAP) is a noninvasive respiratory support tool. It is the gold standard for management of clinically significant obstructive sleep apnea patients [1]. However, it has been reported to be associated with a high prevalence of local nasopharyngeal side effects such as increased neutrophil in the nasal mucosa [2, 3], and it may induce upper airway inflammation [4]. It has been suggested that airway drying or direct distension of nasal mucosa are responsible of the nasopharyngeal inflammation.

Earlier reports had suggested that nonhumidified CPAP has the potential to induce early upper airway inflammation. However, the role of humidified CPAP in this mechanism was not clear. Alahmari et al. [4] used an in vitro model, in which cells were exposed to high pressure to show that drying or the absence of humidification might be responsible for the pro-inflammatory changes that were observed. An experimental study in rats failed to demonstrate any beneficial effects of heated humidification on nasal inflammation [5]. Other clinical and experimental studies have reported positive outcomes on the benefits of humidified CPAP compared with nonhumidified on nasopharyngeal symptoms $[6,7]$.

Most previous in vitro work has used direct distension [8-10], and a mouse model of airway stretch for ventilator-associated lung injury was associated with increased expression of the murine equivalent of interleukin (IL)-8 [11]. Stretch may affect inflammation via oxidative stress because stretch-induced IL- 6 and IL- 8 production can be reduced by antioxidants to increase intracellular glutathione levels [12].

The present investigation aims to examine the impact of humidified CPAP compared with nonhumidified CPAP in healthy subjects on nasal inflammation and upper airway symptoms. Additionally, this study investigates whether induction of nasal inflammation was associated with the development of nasal symptoms after a short period of using humidified and nonhumidified CPAP.

\section{MATERIALS AND METHODS}

Seventeen healthy subjects were recruited. Ethical approval from the Institution's Review Board was obtained, and informed written consent was obtained from all participants.

All participants were free from any nasal symptoms or respiratory diseases. Each participant was evaluated using three different settings. For each setting, a nasal wash sample and nasopharyngeal symptoms using a six-point scale were obtained. First, a baseline measurement without CPAP applications was obtained. Then, after 4 hours, the participant underwent 3 hours of nonhumidified CPAP at $12.5 \mathrm{cmH}_{2} \mathrm{O}$ (REMstar1 Auto M Series with A-Flex ${ }^{\mathrm{TM}}$, Phillips Respironics Inc.,

\footnotetext{
${ }^{1}$ Department of Respiratory Therapy, Faculty of Medical Rehabilitation Sciences, King Abdulaziz University, Jeddah, Saudi Arabia;

${ }^{2}$ Department of Respiratory Care, Prince Sultan Military College of Health Sciences, Dammam, Kingdome of Saudi Arabia;

${ }^{3}$ Department of Clinical Laboratory Science, Prince Sultan Military College of Health Sciences, Dammam, Kingdome of Saudi Arabia

Correspondence: Hajed M. Al-Otaibi, PhD, Department of Respiratory Therapy, Faculty of Medical Rehabilitation Sciences, King Abdulaziz University, P.O. Box 80200, Zip Code 21589, Jeddah, Saudi Arabia; Tel: 00966599914319. Email: halotaibi1@kau.edu.sa
}

Published online at https://www.cjrt.ca on 23 July 2020 
Guildford, UK) through a nasal mask. After 12 days, the participants underwent 3 hours of humidified CPAP (REMstar1 Auto M Series with A-Flex ${ }^{\mathrm{TM}}$, Phillips Respironics Inc.) at $12.5 \mathrm{cmH}_{2} \mathrm{O}$ through a nasal mask. Therefore, there was a 12 -day period between the participants being exposed to the nonhumidified and humidified CPAP levels. Subjects were awake for all procedures. The pressure level of $12.5 \mathrm{cmH}_{2} \mathrm{O}$ was selected because it represents a good high-level pressure that is clinically relevant. During both CPAP settings, the subject's mouth was closed to prevent any possible leakage. All tests were performed in a laboratory where the relative humidity and temperature are kept constant.

\section{Measurements}

\section{Nasal inflammation}

Nasal wash samples were obtained and processed according to a validated protocol that was described elsewhere [13, 14]. Briefly, small pediatric tracheostomy tubes (Bivona Fome-Cuf, size I.D $2.5 \mathrm{~mm}$; Smiths Medical, Kent, UK) were inserted into the participant's nostrils where the nasal lavage was collected. The recovered lavages from the two nostrils were pooled for analysis. All sampling procedures were performed by the same investigator to standardize the procedures. The total portion of the pooled nasal wash fluid from both nostrils was collected in a $15-\mathrm{mL}$ centrifuge tube and processed to analyze the inflammatory mediators and the leukocyte count, as described below. The total pooled nasal wash sample was mixed for 15 seconds on a vortex mixer and then centrifuged for 10 minutes at $2,000 \mathrm{rpm}$ at $4^{\circ} \mathrm{C}$ to yield a cell pellet that was used to determine the leukocyte count. The white blood cell (WBC) count and counts of WBCs that are mononuclear cells (MN; including lymphocytes and monocytes) and polymorphonuclear cells (including all types of granulocytes) was performed using XE 500 model analyzer.

\section{Assessment of nasopharyngeal symptoms}

The presence or absence of nasopharyngeal symptoms (including rhinorrhea, postnasal drip, nasal congestion, sneezing, reduced sense of smell, and itchy nose) were assessed using a six-point nasal score. This assessment was performed before and after the CPAP intervention.

\section{Statistical analysis}

The Kolmogorov-Smirnov test of normality was applied. A one-way repeated measures analysis of variance (ANOVA) with post-hoc Tukey multiple comparison tests were used to examine the differences among baseline, humidified CPAP, and nonhumidified CPAP. Descriptive statistics were used to describe nasopharyngeal symptoms at baseline and after CPAP interventions. A P-value $<0.05$ is considered statistically significant. Data were analyzed using GraphPad Prism 5 software (GraphPad Software Inc., La Jolla, CA, USA).

\section{RESULTS}

Seventeen healthy subjects participated in the study. Measurements were obtained from all participants on three different occasions: baseline (without CPAP), $12.5 \mathrm{cmH}_{2} \mathrm{O}$ of nonhumidified CPAP, and $12.5 \mathrm{~cm}$ $\mathrm{H}_{2} \mathrm{O}$ of humidified CPAP. However, only seven subjects returned for the third measurement of $12.5 \mathrm{~cm} \mathrm{H}_{2} \mathrm{O}$ of humidified CPAP. Table 1 presents the characteristics of all participants.

\section{TABLE 1}

Characteristics of participants who were enrolled into the study. Seven subjects participated in both protocols

\begin{tabular}{lcc}
\hline & \multicolumn{2}{c}{$\mathbf{1 2 . 5} \mathbf{c m H}_{\mathbf{2}} \mathbf{O}(\boldsymbol{n}=\mathbf{1 7})$} \\
\cline { 2 - 3 } Details of subjects studied & Mean & SD \\
\hline Age, years & 25.7 & 5.6 \\
Weight, kg & 86.2 & 21.5 \\
Height, cm & 172.4 & 5.8 \\
Active smokers & $29 \%$ & \\
Nasal symptoms & None \\
Medications & None \\
\hline
\end{tabular}

The repeated-measured ANOVA indicated that there was a significant difference in the inflammatory marker levels among the three different settings. Comparison between nasal wash WBC count $\left(10^{3} / \mu \mathrm{L}\right)$ at baseline, on $12.5 \mathrm{cmH}_{2} \mathrm{O}$ humidified CPAP, and on $12.5 \mathrm{cmH}_{2} \mathrm{O}$ nonhumidified CPAP showed a statistically significant difference $(p=0.016)$. Similarly, comparison between $\mathrm{MN}$ cells $\left(10^{3} / \mu \mathrm{L}\right)$ at baseline on $12.5 \mathrm{cmH}_{2} \mathrm{O}$ humidified CPAP and at $12.5 \mathrm{cmH}_{2} \mathrm{O}$ nonhumidified CPAP also showed a significant difference $(p=0.003)$. Table 2 shows the changes in the inflammatory markers in the nasal wash fluid at the three different settings.

Post-hoc tests showed that there was a statistically significant difference between the nasal wash WBC count $\left(10^{3} / \mu \mathrm{L}\right)$ on $12.5 \mathrm{cmH}_{2} \mathrm{O}$ humidified CPAP and $12.5 \mathrm{~cm} \mathrm{H}_{2} \mathrm{O}$ nonhumidified CPAP $(p=0.03)$. However, the difference between baseline and $12.5 \mathrm{cmH}_{2} \mathrm{O}$ humidified CPAP was not statistically significant. There was also a statistically significant difference in $\mathrm{MN}$ cells $\left(10^{3} / \mu \mathrm{L}\right)$ at $12.5 \mathrm{cmH}_{2} \mathrm{O}$ humidified CPAP compared with $12.5 \mathrm{cmH}_{2} \mathrm{O}$ nonhumidified $\mathrm{CPAP}(p=0.03)$. However, there was no significant difference between baseline and $12.5 \mathrm{cmH}_{2} \mathrm{O}$ humidified CPAP. Figures 1 and 2 show the difference in the WBC count and MN cells at the three different settings.

Changes in nasopharyngeal symptoms occurred in 12 of 17 participants $(70.5 \%)$ in the nonhumidified group. Participants experienced at least one nasal symptom after application of nonhumidified CPAP at $12.5 \mathrm{cmH}_{2} \mathrm{O}$. The overall frequency of all the symptoms in both humidified and nonhumidified CPAP was generally increased. The most commonly observed symptoms were mouth dryness and headache. Table 3 presents the changes in nasopharyngeal symptoms at the three different settings.

\section{DISCUSSION}

This study showed that nasopharyngeal inflammation can be caused by nonhumidified CPAP. The observed signs of nasal leukocyte changes at around $12 \mathrm{cmH}_{2} \mathrm{O}$ of nonhumidified CPAP may support this conclusion. Moreover, the marked changes in nasopharyngeal symptoms after application of nonhumidified CPAP compared with the baseline and humidified CPAP also suggested inflammation. Humidified CPAP showed no signs of early nasal inflammation and was associated with fewer nasopharyngeal symptoms.

The CPAP application was shown to result in early nasal inflammation and an increased number of nasopharyngeal symptoms. Animal experiments showed that application of $10 \mathrm{cmH}_{2} \mathrm{O}$ of nonhumidified CPAP caused nasal inflammation. The authors believe that the mechanism behind these inflammatory changes is mechanical compression of CPAP on the nasal wall and mucosa [2]. Similarly, another animal study showed a direct association between nonhumidified mechanical ventilation and the airway inflammatory process during mechanical ventilation [15]. Alahmari et al. [4] investigated the effect of nonhumidified CPAP application on nasal inflammation in vivo and in vitro. The authors reported that application of $12 \mathrm{cmH}_{2} \mathrm{O}$ nonhumidified CPAP for 3 hours was associated with significant changes in nasal and systemic pro-inflammatory markers [4]. It was also associated with reduced nasal function and an increased number of nasopharyngeal symptoms. Our data are consistent with these findings. Nonhumidified CPAP might be responsible for causing the inflammatory process in the nasal cavity and possibly also in the upper airway. This was shown by the significant changes in leukocyte levels and nasopharyngeal symptoms compared with the baseline. These changes may occur as a result of introducing dry pressure into the nasal cavity.

Humidified CPAP is thought to be one of the best existing tools to prevent, or at least attenuate, CPAP treatment side effects in selected populations [16]. However, there are few investigations on the role of humidified CPAP in early nasal inflammation. Martinez-Vidal investigated the impact of heated humidification on nasal inflammation in animals, and it failed to report any benefits of adding heated humidity to the CPAP system [5]; they concluded that the presence or absence of heated humidification might not be responsible for early nasal inflammation. However, the capability of heated humidification on preventing or at least attenuating the inflammatory effect of CPAP has been 
TABLE 2

Changes in inflammatory markers in nasal wash fluid after CPAP treatment

\begin{tabular}{|c|c|c|c|c|c|c|c|}
\hline \multirow[b]{2}{*}{ Marker } & \multirow{2}{*}{$\frac{\text { Subjects }(n=17)}{\text { Baseline }}$} & \multicolumn{3}{|c|}{ ANOVA } & \multicolumn{3}{|c|}{ Paired $t$-test } \\
\hline & & Humidified CPAP & Nonhumidified CPAP & $P$ value* & Humidified CPAP & Nonhumidified CPAP & $P$ value \\
\hline $\begin{array}{l}\text { WBC count } \\
\left(10^{3} / \mu \mathrm{L}\right)\end{array}$ & $\begin{array}{c}0.0060 \\
(0.0010-0.0250)\end{array}$ & $\begin{array}{c}0.0050 \\
(0.0010-0.0310)\end{array}$ & $\begin{array}{c}0.0370 \\
(0.0060-0.0400)\end{array}$ & 0.016 & $\begin{array}{c}0.0050 \\
(0.001-0.0310)\end{array}$ & $\begin{array}{c}0.0370 \\
(0.0060-0.0400)\end{array}$ & 0.03 \\
\hline $\begin{array}{l}\text { Mononuclear } \\
\text { cells }\left(10^{3} / \mu \mathrm{L}\right)\end{array}$ & $\begin{array}{c}0.0010 \\
(0.0-0.0060)\end{array}$ & $\begin{array}{c}0.0010 \\
(0.0-0.0020)\end{array}$ & $\begin{array}{c}0.0060 \\
(0.0020-0.0190)\end{array}$ & 0.003 & $\begin{array}{c}0.0010 \\
(0.0-0.0020)\end{array}$ & $\begin{array}{c}0.0060 \\
(0.0020-0.0190)\end{array}$ & 0.03 \\
\hline
\end{tabular}

*Data are expressed as the median (IQR).

\section{FIGURE 1}

The difference in WBC count and MN cell levels at three different settings.
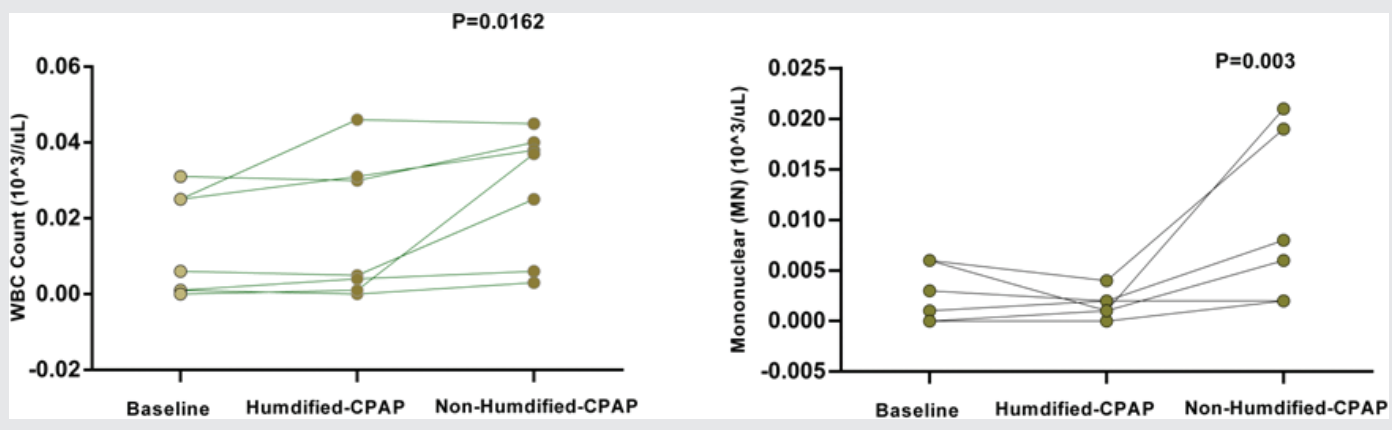

\section{FIGURE 2}

The difference in WBC count and MN cells at two different settings.
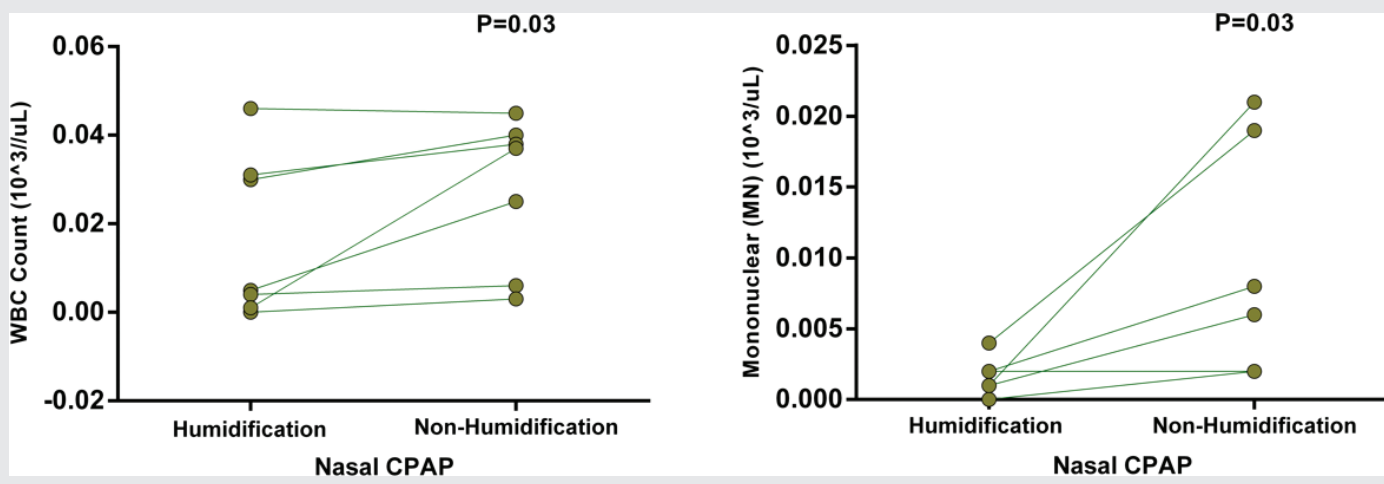

TABLE 3

Changes in nasopharyngeal symptoms

\begin{tabular}{|c|c|c|c|c|c|c|c|c|c|}
\hline \multirow[b]{2}{*}{ CPAP pressure $12.5 \mathrm{cmH}_{2} \mathrm{O}$} & \multicolumn{9}{|c|}{ Nasopharyngeal symptoms, $n$} \\
\hline & Rhinorrhea & $\begin{array}{l}\text { Postnasal } \\
\text { drip }\end{array}$ & Sneezing & Congestion & Insomnia & Itchy nose & $\begin{array}{c}\text { Nose } \\
\text { dryness }\end{array}$ & $\begin{array}{c}\text { Dry mouth/ } \\
\text { throat }\end{array}$ & $\begin{array}{c}\text { Blocked } \\
\text { ears }\end{array}$ \\
\hline Baseline (pre-CPAP) & 0 & 0 & 0 & 0 & 0 & 0 & 0 & 0 & 0 \\
\hline Humidified CPAP & 0 & 0 & 1 & 0 & 0 & 3 & 6 & 4 & 1 \\
\hline Nonhumidified CPAP & 1 & 0 & 5 & 2 & 0 & 9 & 14 & 11 & 6 \\
\hline
\end{tabular}

reported [6]. Adding heated humidification to the CPAP system was shown to at least attenuate the process of nasal inflammation. Applying heated humidified gas during mechanical ventilation would help to avoid detrimental effects on airways that are caused by nonhumidified ventilation [15]. The present data show that adding humidification to the CPAP system would have a significant positive impact on reducing the nasal inflammation process compared with nonhumidified CPAP. Therefore, our data support the use of humidified CPAP because it was associated with significantly lower inflammatory markers. Ryan et al. [17] compared the effect of nonhumidified CPAP, humidified CPAP, 
and application of topical nasal steroids on treating nasopharyngeal symptoms. Ryan et al. found that humidified CPAP is superior to nonhumidified CPAP and topical nasal steroids in reducing the number of nasopharyngeal symptoms after CPAP sessions [17]. Therefore, evidence supporting the use of humidified CPAP as the preferred method for CPAP application is accumulating. Adding heat to the CPAP system might have an additional effect. Our data show that humidified CPAP could attenuate the inflammatory process and it was also associated with fewer nasopharyngeal symptoms. Others reported similar results using heated-humidified CPAP [6, 7]. The superiority of humidified CPAP on heated-humidified CPAP or vice versa warrants further investigation.

Similarly, the present data showed that the number of nasopharyngeal symptoms was markedly reduced as a result of humidified CPAP application compared with nonhumidified CPAP. Others have compared the effect of heated-humidified CPAP and cold, passover humidified CPAP on the nasopharyngeal symptoms. The authors concluded that heated-humidified CPAP was associated with fewer nasopharyngeal symptoms and improved compliance [18]. Other studies showed that adding humidification to CPAP application would reduce the nasopharyngeal symptoms, improve quality of life, and increase adherence to CPAP [19]. Similarly, Koutsourelakis et al. [7] compared the effect of heated-humidified CPAP and heated-sham CPAP on patients with obstructive sleep apnea. The authors found that heated-humidified CPAP was associated with fewer nasopharyngeal symptoms and reduced inflammation markers.

There are several limitations that need to be considered. First, the sample size is relatively small, particularly in the humidified CPAP group. Although a tremendous effort was made to recruit all participants for the third round, only seven participants were able to perform the third session. A larger sample would add more strength to the study and probably improve its generalizability. Second, measurement of serum pro-inflammatory markers would help to draw firm conclusions. Third, the robustness of study design can be improved by adding sham CPAP. However, we have considered each subject as his own control. The present data are consistent with previous reports and suggested the potential role of humidified CPAP in attenuation of the early nasal inflammation process to avoid nasopharyngeal symptoms.

\section{CONCLUSION}

The present investigation suggests that nonhumidified CPAP likely causes early nasal inflammation, and it might be associated with more frequent nasopharyngeal symptoms. We suggest that humidified CPAP should become the gold standard for CPAP system application. Further investigation is needed to compare heat-humidified CPAP with nonheated humidified CPAP.

\section{Funding}

This Project (no. 2014025) was funded by the Deanship of Scientific Research at the University of Imam Abdulrahman bin Faisal University.

\section{Competing interests}

All authors have completed the ICMJE uniform disclosure form at www. icmje.org/coi_disclosure.pdf and declare no conflicts of interest.

\section{Ethical approval}

Ethical approval from the Institution's Review Board was obtained, and informed written consent was obtained from all participants.

\section{REFERENCES}

1. Young T, Palta M, Dempsey J, Skatrud J, Weber S, Badr S. The occurrence of sleep disordered breathing among middle-aged adults. N Engl J Med 1993;323:1230-5. doi: 10.1056/NEJM199304293281704

2. Almendros I, Acerbi I, Vilaseca I, Montserrat JM, Navajas D, Farre R. Continuous positive airway pressure (CPAP) induces early nasal inflammation. Sleep 2008;31:127-31. doi: 10.1093/sleep/31.1.127

3. Lim LH, Wagner EM. Airway distension promotes leukocyte recruitment in rat tracheal circulation. Am J Respir Crit Care Med 2003;168:106874. doi: $10.1164 / \mathrm{rccm} .200207-6900 \mathrm{C}$

4. Alahmari MD, Sapsford RJ, Wedzicha JA, Hurst JR. Dose response of continuous positive airway pressure on nasal symptoms, obstruction and inflammation in vivo and in vitro. Eur Respir J 2012;40(5):1180-90. doi: 10.1183/09031936.00199911

5. Martinez-Vidal B, Farre R, Montserrat JM, et al. Effects of heated humidification on nasal inflammation in a CPAP rat model. Sleep Med 2010;11:413-6. doi: 10.1016/j.sleep.2009.09.010

6. Ruhle KH, Franke KJ, Domanski U, Nilius G. Quality of life, compliance, sleep and nasopharyngeal side effects during CPAP therapy with and without controlled heated humidification. Sleep Breath 2011; 15(3):479-85. doi: 10.1007/s11325-010-0363-2

7. Koutsourelakis I, Vagiakis E, Perraki E, et al. Nasal inflammation in sleep apnoea patients using CPAP and effect of heated humidification. Eur Respir J 2010;37:587-94. doi: 10.1183/09031936.00036910

8. Haseneen NA, Vaday GG, Zucker S, Foda HD. Mechanical stretch induces MMP-2 release and activation in lung endothelium: Role of EMMPRIN. Am J Physiol Lung Cell Mol Physiol 2003;284:L541-7. doi: 10.1152/ajplung.00290.2002

9. Vlahakis NE, Schroeder MA, Limper AH, Hubmayr RD. Stretch induces cytokine release by alveolar epithelial cells in vitro. Am J Physiol 1999;277:L167-73. doi: 10.1152/ajplung.1999.277.1.L167

10. Yamamoto H, Teramoto H, Uetani K, Igawa K, Shimizu E. Cyclic stretch upregulates interleukin- 8 and transforming growth factor-beta 1 production through a protein kinase $\mathrm{C}$-dependent pathway in alveolar epithelial cells. Respirology 2002;7:103-9. doi: 10.1046/j.1440-1843.2002.00377.x

11. Belperio JA, Keane MP, Burdick MD, et al. Critical role for CXCR2 and CXCR2 ligands during the pathogenesis of ventilator-induced lung injury. J Clin Invest 2002;110:1703-16. doi: 10.1172/JCI0215849

12. Jafari B, Ouyang B, Li LF, Hales CA, Quinn DA. Intracellular glutathione in stretch-induced cytokine release from alveolar type-2 like cells. Respirology 2004;9:43-53. doi: 10.1111/j.1440-1843.2003.00527.x

13. Alahmari MD, Sapsford RJ, Wedzicha JA, et al. Intersession repeatability of a novel nasal lavage technique. Transl Res 2011;158:163-8. doi: $10.1016 / \mathrm{j} \cdot \operatorname{trsl} .2011 .04 .001$

14. Hilding AC. Simple method for collecting near-normal human nasal secretion. Ann Otol Rhinol Laryngol 1972;81:422-3. doi: 10.1177/ 000348947208100314

15. Jiang M, Song JJ, Guo XL, Tang YL, Li HB. Airway humidification reduces the inflammatory response during mechanical ventilation. Respir Care 2015;60:1720-8. doi: 10.4187/respcare.03640

16. Lasters F, Mallegho C, Boudewyns A, Vanderyeken O, Cox T, Ketelslagers $\mathrm{K}$. Nasal symptoms in patients with obstructive sleep apnea and their impact on therapeutic compliance with continuous positive airway pressure. Acta Clin Belg 2014;69:87-91. doi: 10.1179/0001551214Z.00000000028

17. Ryan S, Doherty LS, Nolan GM, McNicholas WT. Effects of heated humidification and topical steroids on compliance, nasal symptoms, and quality of life in patients with obstructive sleep apnea syndrome using nasal continuous positive airway pressure. J Clin Sleep Med 2009; 5(5):422-7. doi: $10.5664 /$ jcsm. 27596

18. Massie CA, Hart RW, Peralez K, Richards GN. Effects of humidification on nasal symptoms and compliance in sleep apnea patients using continuous positive airway pressure. Chest 1999;116(2):403-8. doi: 10.1378/ chest.116.2.403

19. Soudorn C, Muntham D, Reutrakul S, Chirakalwasan N. Effect of heated humidification on CPAP therapy adherence in subjects with obstructive sleep apnea with nasopharyngeal symptoms. Respir Care 2016;61(9):1151-9. doi: 10.4187/respcare.04536 